\title{
A 14-bit High Speed 125MS/s Low Power SAR ADC using Dual Split Capacitor DAC Architecture in 90nm CMOS Technology
}

\author{
Chaya Shetty ${ }^{1}$ \\ M.tech Student, Department of \\ Electronics and Communication \\ Engineering, Ramaiah Institute of \\ Technology, Bangalore - 560054, \\ Affiliated to Visvesvaraya Technological \\ University, Belagavi, Karnataka, India \\ e-mail: chayashetty02@gmail.com \\ M. Nagabushanam ${ }^{2}$ \\ Assistant Professor, Department of \\ Electronics and Communication \\ Engineering, Ramaiah Institute of \\ Technology, Bangalore - 560054, \\ Affiliated to Visvesvaraya Technological \\ University, Belagavi, Karnataka, India \\ e-mail: nagabushanam1971@gmail.com
}

\author{
Venkatesh Nuthan Prasad ${ }^{3}$ \\ Assistant Professor, Department of \\ Electronics and Communication \\ Engineering, Ramaiah Institute of \\ Technology, Bangalore - 560054, \\ Affiliated to Visvesvaraya Technological \\ University, Belagavi, Karnataka, India \\ e-mail: nutan2u@gmail.com
}

Received: January 12, 2021. Revised: June 1, 2021. Accepted: June 15, 2021. Published: June 29, 2021.

\begin{abstract}
The proposed work presents a High speed 14-bit $125 \mathrm{MS} / \mathrm{s}$ successive-approximation-register asynchronous analogto-digital-converter (SAR-ADC). A novel-based Dual-Split-ArrayThree-Section (DSATS) capacitor DAC (DSATS-CDAC) is employed to increase the linearity and energy efficiency of the digital-to-analog converter (DAC), additional advantage of this work is that, the area is reduced by $\mathbf{5 9 . 7 6 \%}$ of conventional design. The proposed switching technique of the (DSATS-CDAC) consumes less switching energy. Additionally, bootstrap switching is employed to ensure improved linearity and reduced power consumption.in order to enhance the speed of operation and increase the precision a preamplifier latch based comparator is implemented with the delay of $250 \mathrm{ps}$. The proposed SAR-ADC prototype is implemented in a $90 \mathrm{~nm}$ CMOS process and consumes a power of $42.8 \mathrm{~mW}$ at $1 \mathrm{~V}$ operating supply. The proposed design achieves a figure of merit (FOM) of 37.43 fJ/conversion-step, signal-to-noise-ratio (SNR) of $81 \mathrm{~dB}$, and an effective-number-ofbits (ENOB) of 13.16 bits with a sampling rate of $125 \mathrm{MS} / \mathrm{s}$.
\end{abstract}

Key Words - Successive Approximation Register, Digital to Analog Converter, Dual Split Capacitor DAC, Clock Gated Control Logic, Track and Hold circuit, comparator.

\section{INTRODUCTION}

In the digital world, electronic devices are widely utilized in different fields of science like communication, transportation, etc. In reality, signals being analog in nature need a ADC to make the digital equipment process the analog signals. The fundamental components in these equipment for the inter conversions are ADC and DAC. Recent advancement in applications emphasis on ADC's having high speed, low power and moderate resolution. Earlier flash ADC's were utilized in such applications. But due to the increased number of comparators needed and exponential increase in power consumption with the resolution of the ADC [1][2], flash ADC's became incompetent for such applications. These drawbacks led to studies sorting out that SAR ADCs are highly efficient compared to other ADCs. Within the world of advancing digital technology, performance analyses of digital CMOS circuits are seeing immense improvements. Improvement in the number of features like smaller area, high speed, etc. has led to scaling down of digital circuits. On the other side, the utilization of longer channel length transistor is used even now, in order to achieve improved circuit performance.

Basic signal processing functions like amplification and filtering are often done in a well-oriented format and price effectively [3] using real time analog circuits. Increasing complexity with the advances in electronic systems, implementation in real time analog signals becomes inefficient, impracticable and costlier. So as to permit these electronic systems to interface with real time analog signals, the interconversions of analog and digital signals are essential. This concludes that the key matter of fact for the success of those Electronic systems has always been the advances seen in analog-to-digital converters.

\section{LITERATURE REVIEW}

Globally great efforts are made to develop VLSI systems like ADC's and DAC's that have been one of the most volatile and dominant ones even today in terms of high resolution, power efficiency. This section provides an insight into the research works developed in the last few years to achieve better converter design. Q. Fan and J. Chen, [4] Built a digital-slope 13-bit SAR-Assisted-Time-Interleaved ADC. Here a course and fine ADC's are cascaded to get high resolution and using charge sharing methods to eliminate large power consumption on a chip, the design achieves an SNDR of $63.74 \mathrm{~dB}$ and FOM of 3.83fJ/conv. K. Kuo, [5] Implemented a Binary Search 10bit and two SAR ADC's with a two-bit upgrade to Error Tolerance Capabilities. Polineni, S., Bhat, M.S. \& Rajan [6], proposed a 10-bit Enhanced MSB Capacitor-Split Switching Technique is used in the fully differential switching scheme for Ultra-Low-Power Enhanced. The proposed design is energy- 
efficient and high precision. The switching scheme is energy efficient at $96.88 \%$ and capacitor area-efficient at $50 \%$ compared to the conventional scheme. The switching scheme features make it ideal for low-power and high-resolution ADC realization. Mao, W., Li, Y., Heng, C.H. and Lian, Y [7]. They describe a multi-segmentation-digital to analogue converter architecture that uses a hybrid switching technique to minimize the overall number of unit capacitors for biological signal processing, SAR ADC is widely used. The NFBWA DAC and hybrid switching method will reduce the DAC part's total unit capacitor count by $87.4 \%$. The results measured show that the ADC achieves 10.47 ENOB and absorbs 110-nW power. Maddox, M. C. W. Coln, Y. Lu, and L. D. Fernando, [8] addresses a high precision converter, it solves the sharing of passive charges by SAR ADC. A passive charge sharing design with one reference cap per bit and a short switch is proposed for SAR ADC, as well as a calibration method that solves the steps needed to attain 16-bit linearity. M. Kulkarni, C. Parikh, and S. Sen, [9] demonstrated a Systematic Approach for Determining Capacitors' Weights in Non-Binary Redundant SAR ADCs' DAC. The analysis brings out that the non-binary redundant SAR ADC provides SNR enhancement along with the speed boost. The proposed algorithm addresses the weights of the capacitors used in the DAC. That weight may be used in both synchronous and asynchronous SAR logic irrespective of the DAC switching scheme. S. Kim and K. Kwon, [10] Presented a hybrid ADC combining capacitive multi-bit / cycle DAC-based SAR ADC with Flash ADC. The design achieves a speed boost and the CDAC capacity size is $2 \mathrm{pF}$ which is a significant reduction from a conventional CDAC. Hybridization reduces the data conversion time from $390 \mathrm{~ns}$ to $140 \mathrm{~ns}$.W. Kim et al. [11]. A Low Noise Asynchronous SAR-Assisted Time-
Interleaved SAR (SATI-SAR) ADC was implemented. The SWC operation reduces the sizes of the logic blocks in the SAR control loops, along with saving power-consumption. The Architecture uses a CDAC to improve the linearity of 12 bit. Zhang, Dai, and Atila Alvand pour [12] describe a 14-bit 10$\mathrm{KS} / \mathrm{s}$ Successive-Approximation-Register which converts analog to digital form (ADC) for biomedical applications. A uniform geometry, a non-binary-weighted-capacitive digitalto-analog-converter is implemented to achieve enhanced linearity, and error correction is done using dynamically shift decision levels for the secondary-bit approach. Chen, C-H., Y. Zhang, J. L. Ceballos, and G. C. Temes. [13] Presented a SAR ADC Noise shaped with three capacitors. The literature proposes an effective SAR ADC. Application of oversampled schemes will achieve a high SNQR. In ultra-deep submicron CMOS technologies, the output of the proposed noise-formed ADC will be less degraded by the leakage current for lowfrequency operation than for existing converters. C. Liu, S. Chang, G. Huang, and Y. Lin, [14]. A 10-bit 50-MS/s SAR ADC was constructed with Monotonic Capacitor Switching Procedure. High resolution converters are implemented using the cascaded/hybrid switching/ fully differential design with very high complex circuitry and large power consumption where a fully differential design is better in terms of complexity and speed

A completely differential ADC requires a larger capacitor array and typically consumes more space. Despite the fact that the unit capacitor size can be almost halved in fully-differential designs, the layout size of the dual capacitor array is typically larger, despite the fact that the dummy structures inside do not scale well with the unit capacitor size.

TABLE I. SUMMARY OF LITERATURE REVIEW

\begin{tabular}{|c|c|c|c|c|c|c|c|c|}
\hline Reference Journals & [4] & [5] & [6] & [7] & [8] & [11] & [13] & {$[14]$} \\
\hline $\begin{array}{c}\text { Process } \\
\text { Technology }(\mathrm{nm})\end{array}$ & 28 & 90 & 180 & 130 & 55 & 55 & 55 & 55 \\
\hline $\begin{array}{c}\text { DAC } \\
\text { Architecture }\end{array}$ & $\begin{array}{l}\text { Switched } \\
\text { DAC }\end{array}$ & $\begin{array}{l}\text { Capactive } \\
\text { DAC }\end{array}$ & $\begin{array}{l}\text { Switched } \\
\text { DAC }\end{array}$ & $\begin{array}{c}\text { Binary } \\
\text { Weighted } \\
\text { CDAC } \\
\end{array}$ & $\begin{array}{c}\text { Charge } \\
\text { Sharing } \\
\text { Capactive DAC } \\
\end{array}$ & $\begin{array}{l}\text { Split } \\
\text { CDAC }\end{array}$ & CDAC & CDAC \\
\hline Resolution (bits) & 13 & 10 & 11 & 12 & 16 & 16 & 16 & 16 \\
\hline $\begin{array}{l}\text { Sampling } \\
\text { Rate }(\mathrm{S} / \mathrm{s})\end{array}$ & $500 \mathrm{M}$ & $250 \mathrm{M}$ & $2 \mathrm{~K}$ & $1 \mathrm{~K}$ & $1 \mathrm{M}$ & $1 \mathrm{M}$ & $1 \mathrm{M}$ & $1 \mathrm{M}$ \\
\hline ENOB & - & 8.645 & 10.14 & 10.47 & - & - & - & - \\
\hline FOM (fJ/conv) & 3.83 & 77.3 & 120 & 76 & 0.126 & 0.126 & 0.126 & 0.126 \\
\hline SNR (dB) & 63.74 & - & 62.8 & 64.8 & 100 & 100 & 100 & 100 \\
\hline
\end{tabular}

There have been several methods offered to minimize the size of a standard fully-differential-capacitor array without using digital-calibration techniques $[6,13]$, but each has its own set of limitations. The size of the capacitor-array is reduced using the top-plate-sampling technique in [6]. As a result, the comparator's common mode is input dependent, and there's a chance of higher non-linearity errors.

The bottom-plate-sampling approach is kept while the array of capacitors is reduced to half its original size in [13]. Nonlinearity mistakes may occur as a result of the approach used. Although the completely differential topology has received a lot of attention in recent publications for various applications,

Due to the power and area limits for biomedical applications, the dual split capacitor DAC with differential sampling and single ended SAR ADC was chosen. Extra design time and energy are required to design and optimize for low power, hence a D flip-flop is utilized as a basic cell in the SAR logic. The only attractive choice for medium resolution and low power is the SAR ADC converters; these approaches provide better power efficiencies. The literature summary in Table-I shows that the proposed converters use conventional type of switching schemes to reduce the power consumption as with 
different node technologies the switched CDAC is implemented but with increase in resolution and higher sampling rates the drawbacks are the achieved SNR is lower and figure of merit (FOM) is very low hence giving rise to nonlinearity of the converter which puts a limit on resolution and high speed of operation, Several strategies have been applied to maximize the area and power efficiency in the suggested work, which focuses on a Dual split differential architecture with bootstrap sampling, in which multiple strategies have been used to optimize the area and power efficiency as follows:

A dual split capacitor DAC is developed for reduction in total area of the capacitance by more than $50 \%$ and a multiplier based switching is adopted for low power and also resulting in reduction in the switching energy.

- A preamplifier latch-based-comparator is designed and the circuit is controlled by asynchronous SAR logic for high speed and low dispersion delay with supply of $1 \mathrm{~V}$ which eliminates external clock and also results in the optimized power of the comparator.

- To improve SNR an optimized bootstrap switch is used for sampling and using it in the differential mode with two complementary clocks for operation, a high speed, high SNR up to $80 \mathrm{~dB}$ can be achieved.

The main goal of the proposed paper is to improve sampling rate higher than $100 \mathrm{MS} / \mathrm{s}$ compared to the literature and to achieve further improvement in the SNR, ENOB, FOM and to present a DSATS-based DAC methodology to save switching energy and increase DAC linearity as compared to conventional architecture.

The objective of the proposed design is obtained from the literature summary and the specifications for the proposed design to meet the required resolution and 14-bit accuracy are addressed in Section II. Section III covers the ADC's subblocks, including bootstrap switching, the capacitive DAC's proposed design with multiplexer switching, and a preamplifier latch-based comparator with clock generating circuitry and SAR logic. Section IV discusses the complete integration of all the sub blocks. Section $\mathrm{V}$ discusses the proposed ADC structure's simulated measurement findings and, finally, the conclusion is presented in section VI.

\section{SAR-ADC KEY BUILDING BLOCKS}

The full SAR ADC architecture is depicted in Fig. 1 as a conceptual design. Differential sampling circuit, dual-split capacitor DAC design with switches, capacitors, voltage comparator and digital SAR logic are all part of it. This composition perfectly aligns the converter with the primary goal of optimizing the SAR logic and as a result, the converter's performance. The unit capacitance is $8 \mathrm{fF}$ is kept as tiny as possible to save space and switching energy with the total capacitance of DAC is roughly $824 \mathrm{fF}$ in this dual split DAC combination. Finally, the preamplifier latch-based voltage comparator is responsible for the SAR logic's operation. The inputs to SAR logic are driven to zero via a binary search at the start of conversion, and the input voltage is sampled on the capacitive DAC. The comparator makes its first judgment based on the polarity of the sampled output of the sampler, which reflects the most significant output bit (MSB). Depending on the result of this decision, to move the comparator input toward zero, the CDAC's greatest capacitance is switched. This process is repeated in smaller phases until all bits are resolved at the completion of the conversion, at which point the DAC output is valued. A small sampling capacitor is used for higher SNR and to achieve rail-to-rail signal swing. The complete design implementations of all the sub-modules are discussed in the next preceding sections

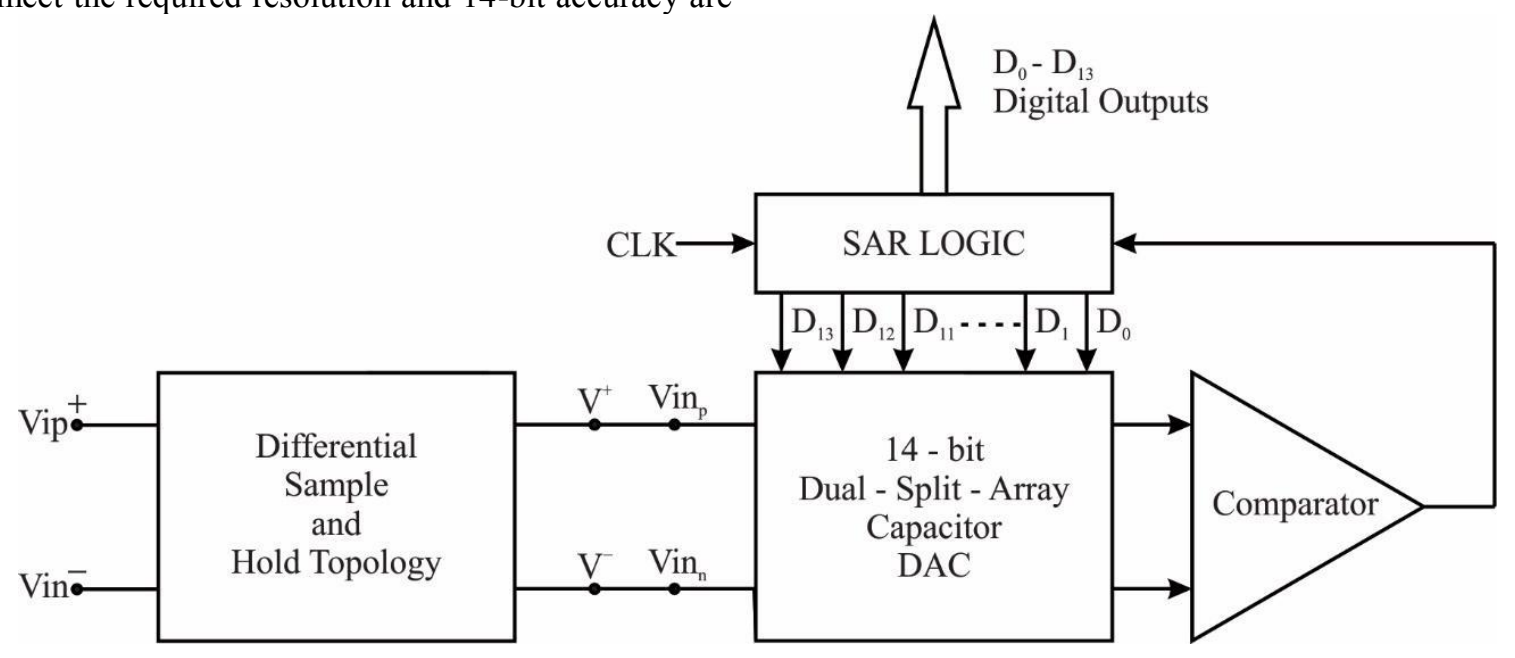

Fig. 1. Complete SAR ADC Architecture Built with Sub-Modules 


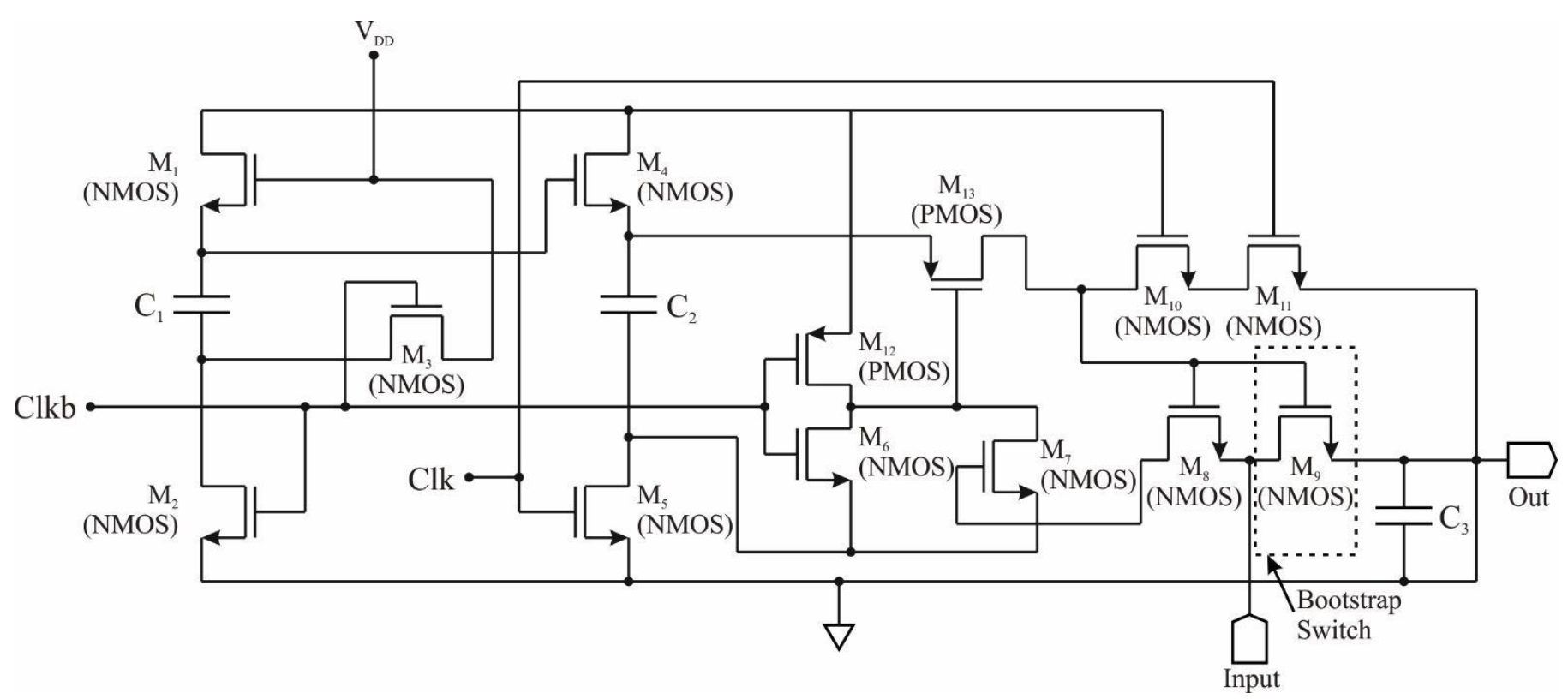

Fig. 2. Bootstrapped Switch

\section{A. Differential Sampling Circuit}

A fully differential architecture is one of the best methods to suppress supply noise and improve common-mode-noiserejection and to achieve a 14-bit accuracy. The conventional Sample and hold $(\mathrm{S} / \mathrm{H})$ circuits were widely used for lowresolution data converters with resolution less than 8-bits and these conventional circuits [15] [16] suffer from many drawbacks like non-linearity, low speed, large capacitance. These circuits are seldom used in high-resolution, high-speed data converters. The proposed bootstrap topology has proven to be one of the best robust topologies for high speed, high resolution, and better linear characteristics.

\section{B. Design of Bootstrap Switch}

Bootstrapping switches are very power efficient due to the reason that there is no static power consumption while switching operation, and the parasitic body diodes on the chip are not active during the entire operation in sample and hold modes, bootstrapping switches are extremely energy efficient. The dynamic switching activity of the circuit is mostly responsible for the power loss in the bootstrap switch [17].

Fig. 2 shows the proposed bootstrap switch circuit, where the transistors M1-M5 are responsible for generating a gate voltage that is shifted by the NMOS transistor M8, C2, and the PMOS transistor M13 concerning the input voltage. When Clkb goes high, the NMOS M6 turns ON and applies the input voltage to the drain of PMOS M12, which drives the NMOS M7 to generate the sampled output voltage. The proposed design requires a single clock to attain a steady-state and the capacitors $\mathrm{C} 1, \mathrm{C} 2$, and $\mathrm{C} 3$ are charged to supply voltage at a steady state [18]. The charge across the output capacitor $\mathrm{C} 3$ is not larger than the supply voltage, so the driving voltage for the transistor switch does not exceed the supply voltage. The bootstrap switch samples the input voltage up to the supply voltage. With the bootstrapped switch M9, the gate-source voltage of the sampling transistor M8 is fixed at the supply voltage, resulting in a smaller constant on-resistance and better switch linearity. The dimensions of transistors $(W / L) p$ and $(W / L) n$ are the default values that are available in gpdk $90 \mathrm{~nm}$ CMOS technology in the cadence virtuoso schematic editor tool. The design value of NMOS and PMOS is given in Table II.

TABLE II. Design Values for BoOtSTRap SWitch

\begin{tabular}{|c|c|}
\hline Parameters & Value \\
\hline PMOS: $(W / L)_{1,2,3}$ & 1.2 \\
\hline NMOS: $(W / L)_{1,2,3,4,5,6,7,8,9,10}$ & 1.2 \\
\hline
\end{tabular}

Fig. 3 shows the simulation characteristics of the proposed Bootstrap Switch. The proposed bootstrap topology samples the input signal at $10 \mathrm{MHz}$ frequency, with a sampling clock rate of $125 \mathrm{MS} / \mathrm{s}$ i.e. at $8 \mathrm{~ns}$ of the clock period, the sampling voltage range varies from $1 \mathrm{mV}$ to a maximum of $1000 \mathrm{mV}$ without any distortion at an output voltage as shown in Fig. 3 


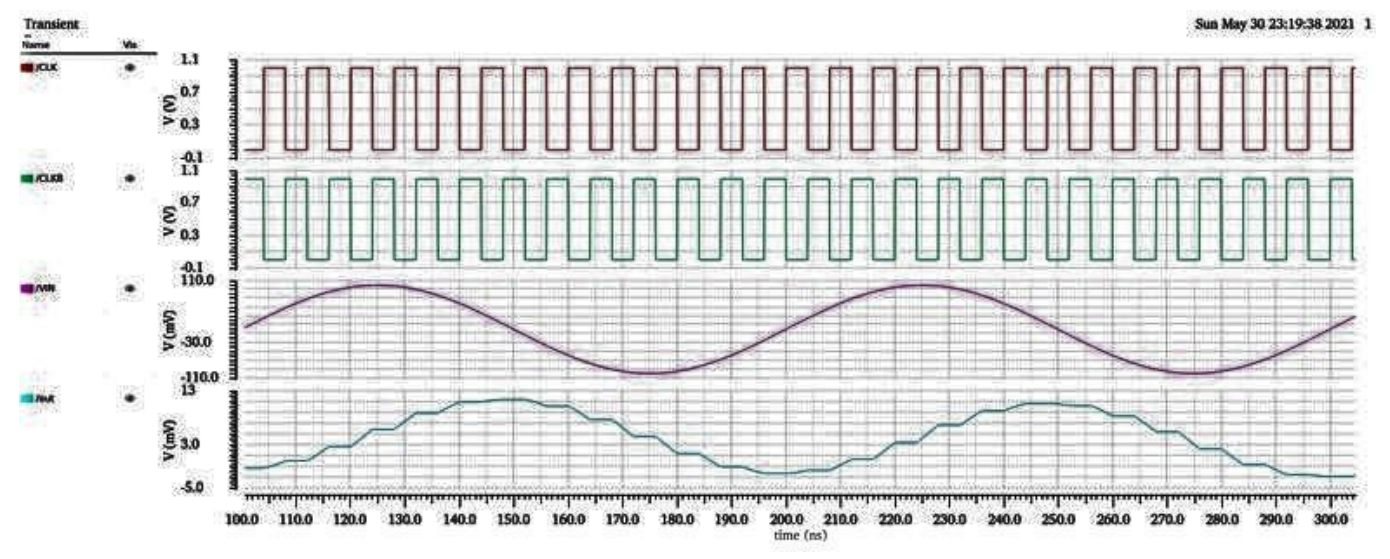

Fig. 3. Simulation Characteristics of Proposed Bootstrapped Switch

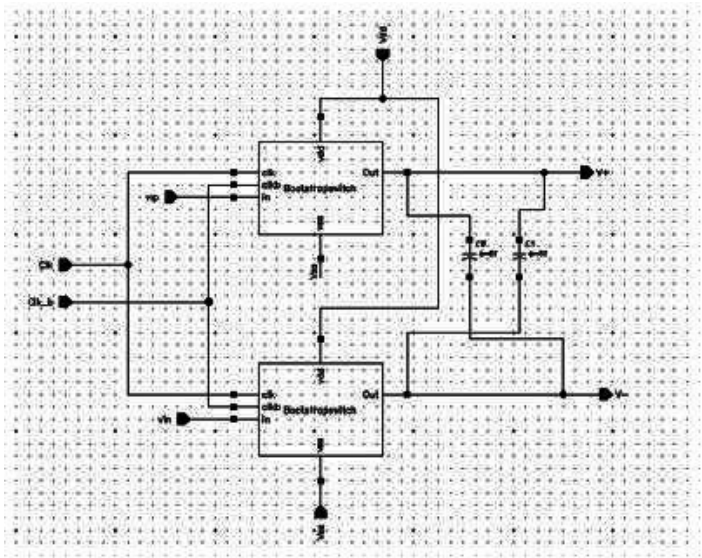

Fig. 4. Proposed Differential Samples and Hold Topology

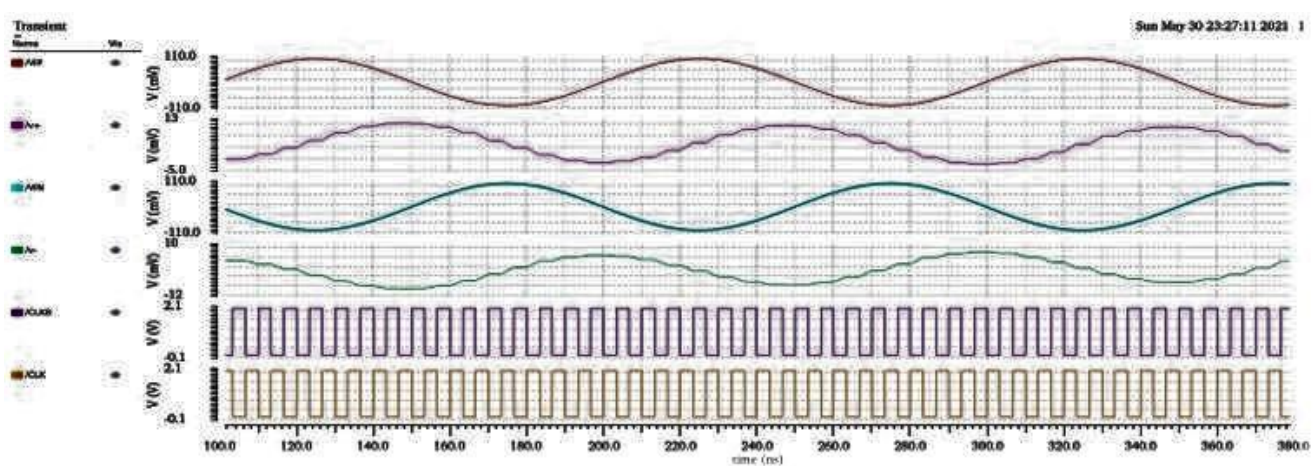

Fig. 5. Simulation Characteristics of Differential Sample and Hold Topology

\section{Proposed Differential Sample and Hold Topology}

The proposed bootstrap switch in section 3.2.1 is used to develop a differential [19] topology by arranging in differential mode as shown in Fig. 4, the two bootstrap switches are coupled using cross coupling capacitors to generate the differential output, When the bootstrapped switch is turned off, the input signal couples with the sampling capacitors via the Cds formed by the sampling transistor M8 the MOS drain-source capacitor and the routing parasitic capacitance. This coupling effect [20][ 21][22] corrupts the high-frequency execution, due to the unequal charges generated by $\mathrm{Cds}$, resulting in a complex offset, to further reduce these adverse effects the capacitors are cross-coupled at the output of differential sample \& hold topology.

The proposed differential sample and hold [23] topology is simulated with an input signal of $10 \mathrm{MHz}$ frequency, with sampling complimentary clocks at $125 \mathrm{MS} / \mathrm{s}$ and simulated with the complimentary sampling input voltages [19] [22] varying between $1 \mathrm{mV}$ to a maximum of $1000 \mathrm{mV}$ as shown in Fig. 5, the complementary output obtained are digitized version of input signal with an advantage that there is a reduction in distortion and improved SNR compared to single-ended type topologies. 


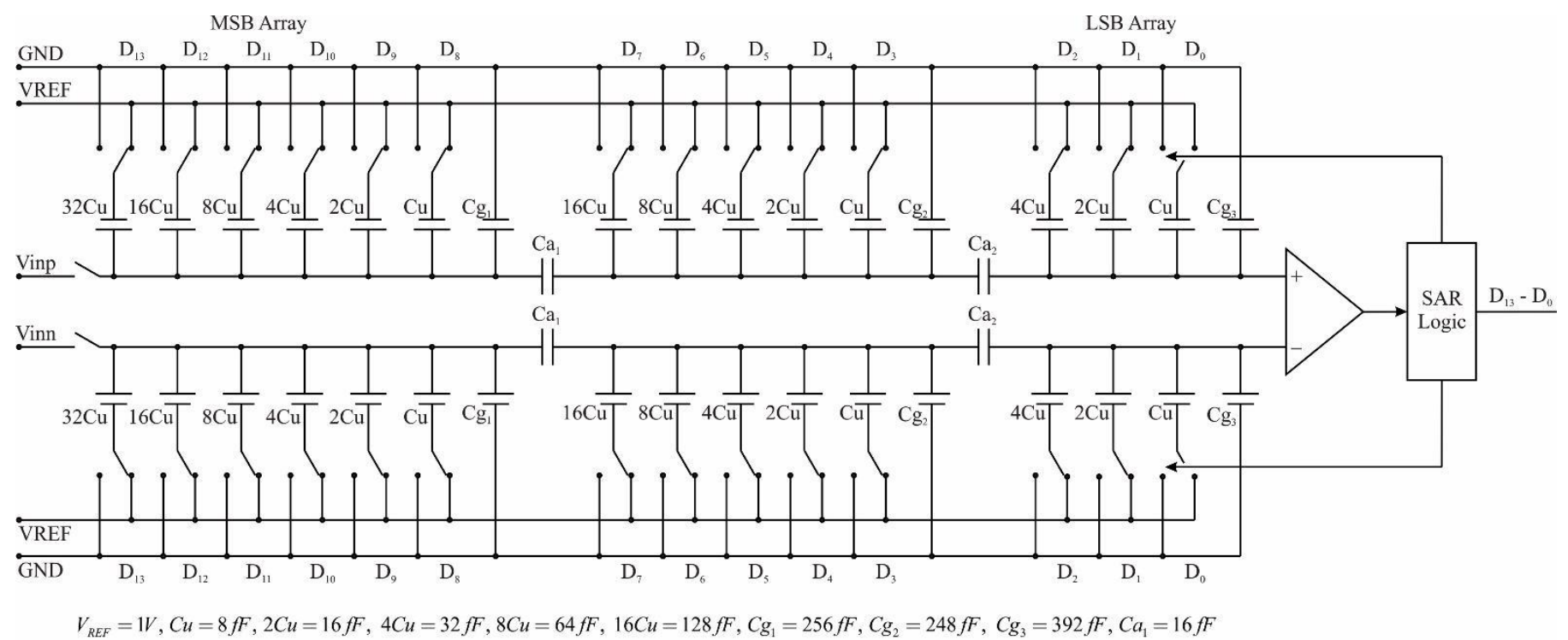

Fig. 6. Complete 14-bit Dual Split Capacitor DAC Architecture Circuit

\section{Dual-Split-Capacitor-based-DAC Design Structure (DSC- $D A C)$}

In the conventional design and development phase of any SAR ADC [24] the crucial building block is the DAC architecture, which has the greater importance compared to other building blocks due to the reason that the major area and power consumption in an $\mathrm{ADC}$ is because of the DAC module. Hence the total area and the power consumption is taken care by optimizing the design to the great extent. The main source of energy consumption in the capacitive DAC is during charging, therefore understanding the capacitor architecture is very vital. In a conventional binary-weighted capacitive DAC, the number of capacitors [25] required and the area occupied is very large, if the required resolution is 14 bits, in such cases, the MSB capacitor needs to be $214 \mathrm{Cu}$, where $\mathrm{Cu}$ is unit capacitor then MSB capacitor will have a larger value in the Pico- farad range thus occupying large area and power, to overcome such disadvantage the split architecture is proposed. To achieve a 14- bit accuracy, to suppress supply-noise and improve common-mode-noise-rejection a fully-differential (DSATS -DAC) architecture is proposed. Where the MSB capacitor required is $25 \mathrm{Cu}$, with the measure of MSB capacitance being $256 \mathrm{fF}$ which is very small compared to the conventional architecture, saving a large amount of area.

The proposed DAC architecture is a modified version of a conventional single split capacitor DAC architecture called dual split capacitor DAC that eventually reduces the total area capacitance by $99 \%$. The existing DAC architectures for low power applications are capacitor-based DAC architecture which are better due to the zero quiescent current and capacitor mismatch required are very less compared to resistor type DAC structures [26] [27]. The matching requirements can be further reduced using complex digital circuitry, these complexities can be further reduced with improved speed performance by proposing the dual split array architecture with $\mathrm{Ca} 1$ and $\mathrm{Ca} 2$ as shown in Fig. 6. The proposed design of using the dual split capacitor DAC architecture is shown in Fig. 6.

In this research work, the capacitor array is divided into two separate arrays [28] and three segments, L, M, and N, with lengths 6,5 , and 3 that satisfy the required resolution as shown in fig.6. Let us consider $\mathrm{Ca} 1$ and $\mathrm{Ca} 2$ to be the two split capacitors with ground capacitances $\mathrm{Cg} 3$ and $\mathrm{Cg} 2$ and these capacitances can be evaluated considering the expression (1) and (2), where $m$ and $n$ are the length of the sections. Q is the least value of the capacitor ratio in the adjacent section, i.e $Q=1$. $\mathrm{Cu}$ is the unit capacitance. The unit capacitance $\mathrm{Cu}$ is calculated by considering the sampling rate as $125 \mathrm{MS} / \mathrm{s}$ with $N=14$ bit resolution, few of the possible significant combinations of L, M, $N$ segments can be depicted using expressions (3), (4), (5), (6).

$$
\begin{aligned}
& \frac{c_{g 3}}{c_{u}}=\left(\frac{2^{m}-1}{Q}\right) *\left(\frac{c_{a 2}}{c_{u}}\right)-2^{m}-1 \\
& \frac{c_{g 2}}{c_{u}}=\left(\frac{2^{n}-1}{Q}\right) *\left(\frac{c_{a 1}}{c_{u}}\right)-2^{m}-1
\end{aligned}
$$

Considering

$$
\begin{gathered}
\frac{c_{a 1}}{c_{u}}=w, \frac{c_{b 2}}{c_{u}}=x, \frac{c_{a 2}}{c_{u}}=y, \frac{c_{b 3}}{c_{u}}=z \\
y=2^{m} \\
z=\frac{4^{m}}{Q}-2^{m+1}+1 \\
x=\frac{\left(2^{n}-Q\right) * w}{Q}-\left(2^{n}-1\right)^{*} Q
\end{gathered}
$$


With resolution of 14 bits, capacitors are divided into the segments with length $L=6, M=5$ and $N=3$, The capacitor ratio is taken as unity. With these considerations, the total capacitance obtained for DSATS-CDAC is $824 \mathrm{fF}$, with unit capacitance calculated to be $8 \mathrm{fF}$, whereas for conventional binary weighted CDAC, it is $824 \mathrm{pF}$, saving large amount of area around $90 \%$, the switching energy for the proposed design is $8 \mathrm{fJ}$ whereas for the conventional binary weighted CDAC it is 50fJ for the same specification, these values illustrate that the proposed DAC is energy and area efficient. The operation of differential DSATS-CDAC consists of two sections namely the upper section and the lower section, when vinp, vinn is applied to the bootstrap circuit the sampled signal serves as the input on to the upper DSATS-CDAC top plate, whereas the bottom plate is reset to VREF. The comparator now performs comparison at its inputs, MSB bit in the SAR register is set then $32 \mathrm{Cu}$ is connected to GND, similarly the operation is repeated by connecting $16 \mathrm{Cu}$ to VREF and the cycle is repeated until the LSB bit is decided and all the 14-bits are set/reset in SAR register. This sort of DAC operation is referred to as monotonic since only one capacitor switch is used for each bit cycle, reducing charge transfer and resulting in lower power dissipation [29].

\section{E. Switching Comparator Design}

The comparator plays an important role in decision making and its switching function is the limiting component in highspeed data converter design, due to its power consumption, accuracy, and comparison speed [30]. The design of the comparator is a part of the complete system and is analog in nature. Comparator is one of the critical components in the implementation of the Successive Approximation Register $\mathrm{ADC}$ which is responsible for the conversion speed [31]. Because of its finite precision, comparison speed, and power consumption, the comparator is critical in the design of a highspeed data conversion system.

In the proposed work, for a $14 \mathrm{bit}$ SAR ADC, a preamplifier latch-based comparator is proposed that suits the basic requirements as it allows high-speed and low-powerconsumption as shown in Fig. 7. The MOS transistors M1-M5 constitute the pre-amplifier stage acting as input stage that amplifies the smallest analog signal that can be applied to comparator, where the NMOS transistor M5 acts as a bias transistor with $\mathrm{W} / \mathrm{L}$ as $50(5 \mu / 100 \mathrm{n})$. The preamplifier circuit also acts as the current mirror circuit with two PMOS transistors M1 and M2 having an aspect ratio (W/L) of $3.6(360 n / 100 n)$, the NMOS transistors M3 and M4, with an aspect ratio (W/L) of $1.5(150 \mathrm{n} / 100 \mathrm{n})$ is used to provide dc offset voltage. The MOS transistors M6 -M9 forms the latch stage which is responsible for decision making and generating the output. In the latch stage, the PMOS transistors M6 and M9 and the NMOS transistors M7 and M8 are connected in anti-parallel to reduce the MOS transistors secondary effects, with the aspect ratio of $1.5(150 \mathrm{n} / 100 \mathrm{n})$. The output of the preamplifier is taken as an input to the second stage PMOS transistors and as the internal clock goes high the comparator operation is initiated.

The feedback from the latch stage to the preamplifier stage reduces the noise in the signal [32]. The output is then converted into a logical signal by the output buffer formed by the transistor M10-M14 with W/L of $1.5(150 \mathrm{n} / 100 \mathrm{n})$, which further reduces the offset voltage. The final comparator output obtained is free from noise variations. The combined preamplifier and latch stage ensures the high-speed operation and low power dissipation [33]. The high precision and resolution is obtained due to the amplifier being able to reduce the offset voltage. Fig. 7. Shows the schematic view of the high-speed differential comparator design.

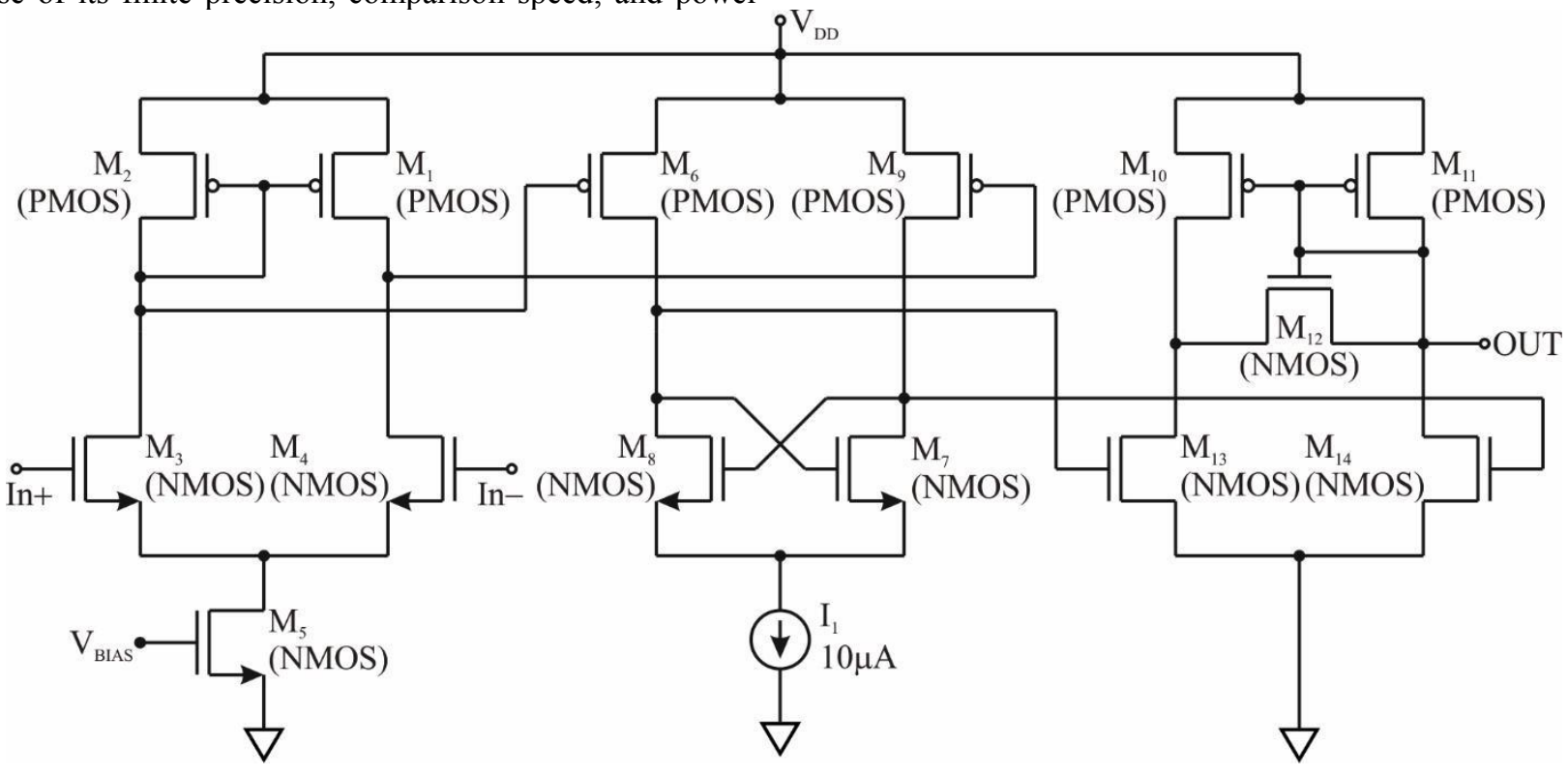

Fig. 7. Preamplifier Latch based Comparator Circuit 


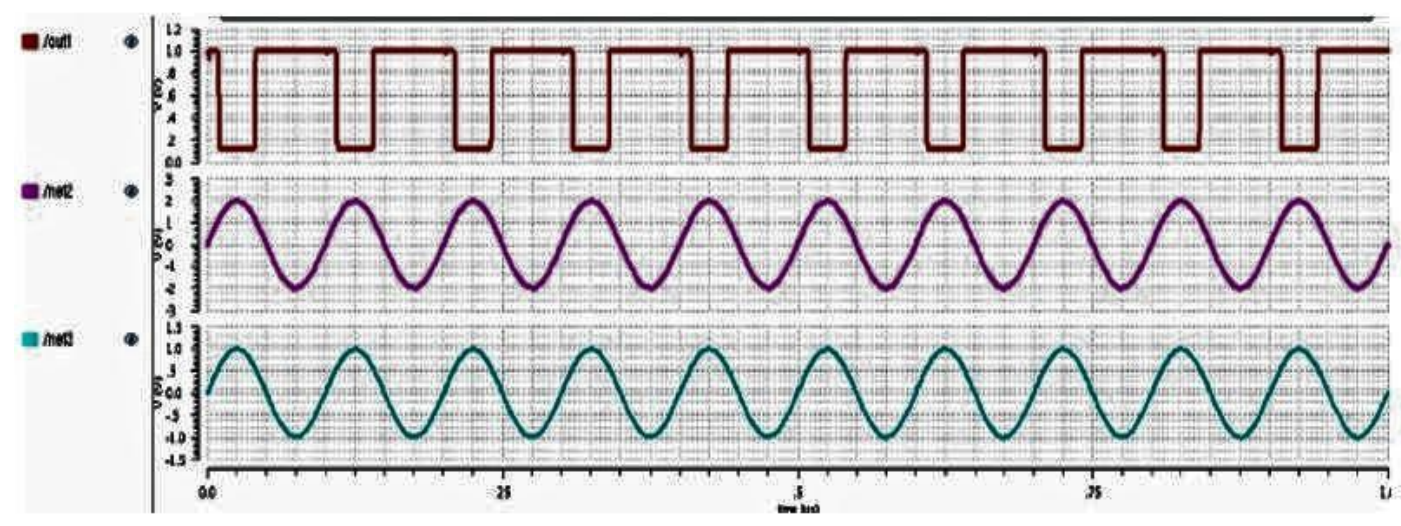

Fig. 8. Simulation Result of Preamplifier Latch Comparator

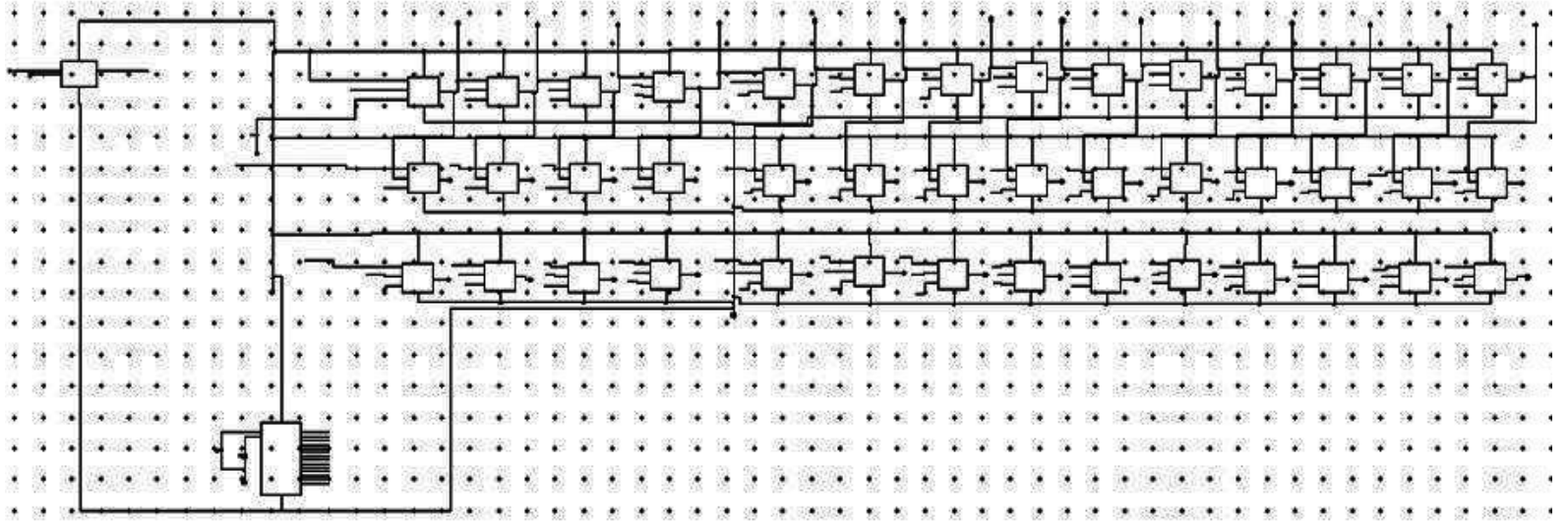

Fig. 9. Circuit Diagram of SAR Logic

The proposed preamplifier latch-based comparator with its simulation results for high-speed and low power-consumption are as shown in Fig. 8. The topology is simulated with a $1 \mathrm{~V}$ power supply and applied input sampling frequency of $10 \mathrm{MHz}$ keeping Vbias $=0.5 \mathrm{~V}$ and Ibias $=10 \mu \mathrm{A}$. The simulation results of the propagation delay variation versus the input overdrive voltage with varying common-mode voltage are measured, as shown in Fig-8. With the simulated results it shows that the delay dispersions of the proposed comparator are $250 \mathrm{ps}$.

\section{F. The SAR Logic}

The SAR logic block architecture is the core control circuit for the data converter as it is one of the most crucial decision blocks in making the complete logic system work. This logic block works similar to the digital weighing system, the output digital bits [23] for the applied analog input signal value and switching signals are sent to the DAC at proper intervals of time to obtain an approximated digital word within the conversion time of the converter. Successive registers use a binary search method with control logic for analog conversion [34]. DSATSDAC samples the input signal at the start of the clock during the sampling phase. Based on the charges stored in the DAC, the differential input at the comparator switches to logic 1 or to logic 0 . The change in the comparator inputs is due to the stored value and the approximated analog output of the DAC. The Successive register stores $\mathrm{N}$ bits of binary data for an N-bit converter based on the set/reset state of the register as shown in Fig. 9. The set bit can be a logic ' 1 ' whereas the reset is ' 0 ' or vice-versa. In the first stage of the 1 st clock pulse, the rightmost register bit is set to ' 1 ' and other registers are reset to ' 0 '. Similarly, the other N-1 bits of the N-bit converter are determined by a similar process. The process repeats for 14 clock cycles, at the end of the 14th cycle the approximated register holds the N-bit binary word. Thus, for an N-bit ADC N clock cycles are required to convert analog to digital word.

The NAND gates are utilized in conjunction with the D-flipflop to supply the desired bit [35]. The chosen signal decides the DAC switch management signals and stores the output binary word through the Approximation registers as shown in Fig. 11. This kind of optimized SAR management logic makes single bit conversion easy without any additional delay [36] time between the latch comparator output and the DAC management signals, so that a better sample rate can be achieved. NAND gates and D-flip-flop are utilized to supply the sequence shifter. For low power converters the NAND gates are used to guarantee that one clock pulse is received by D-flip-flop as shown in Fig. 11(b). 
Fig. 10. Circuit Diagram of Clock Generation Circuit for SAR Logic

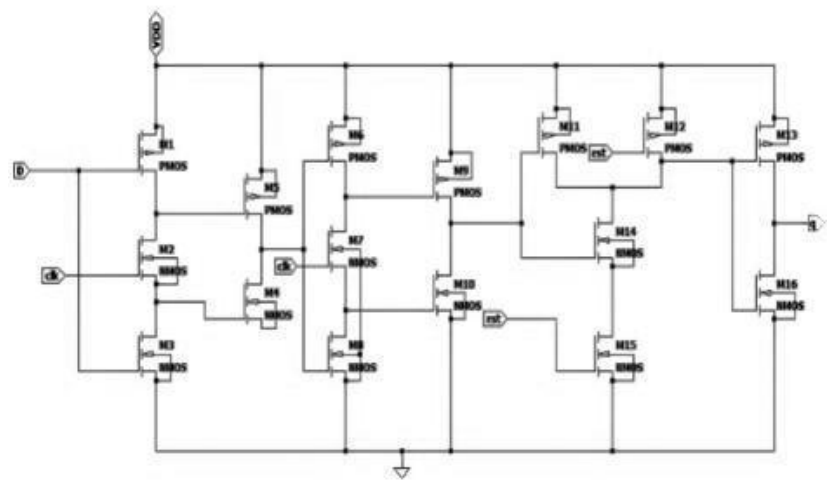

(a) Circuit Diagram of D Flip-Flop

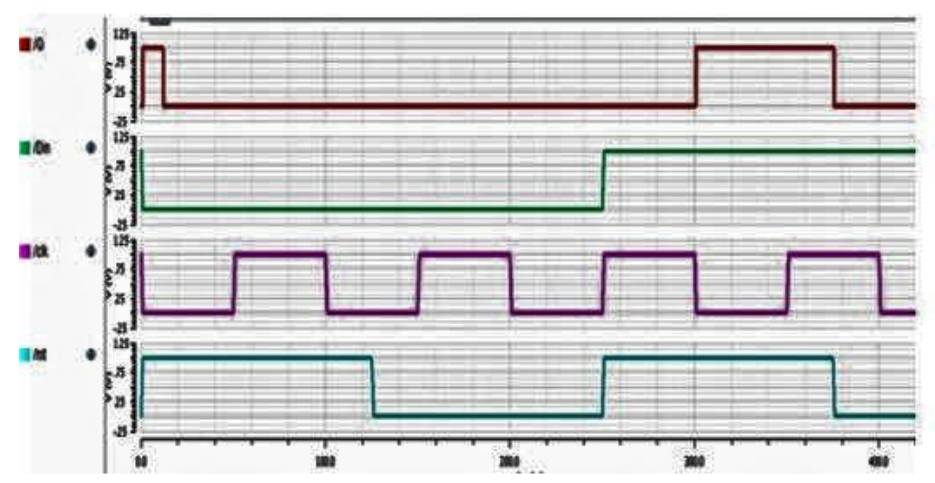

Fig. 11. (b) Transient Response of the D Flip-Flop

\section{INTEGRATION OF 14-BIT SAR ADC}

The complete 14-bit SAR ADC is implemented using submodules as discussed in the previous sections and by aggregation of all the sub-modules a complete 14-bit SAR ADC is constructed. The complete VLSI design is implemented in 90nm CMOS technology as shown in Fig. 12. Using the Cadence EDA simulation environment for a confirmative block [37] level steady state analysis and verification using the long transient simulations are employed to characterize the parameters of the complete design module as shown in Fig. 13, the transient response in Fig. 13 shows during every clock cycle the SAR-register bits are set/reset and at the end of 14th clock, all the 14-word bits are available at the output [38] of the SAR. When the transient response progresses in time, there is an incremental shift in steps, which is the approximated digital output of proposed SAR [39] [40]. Analyzing the results obtained from the simulations, the key characteristic performance parameters are evaluated. 


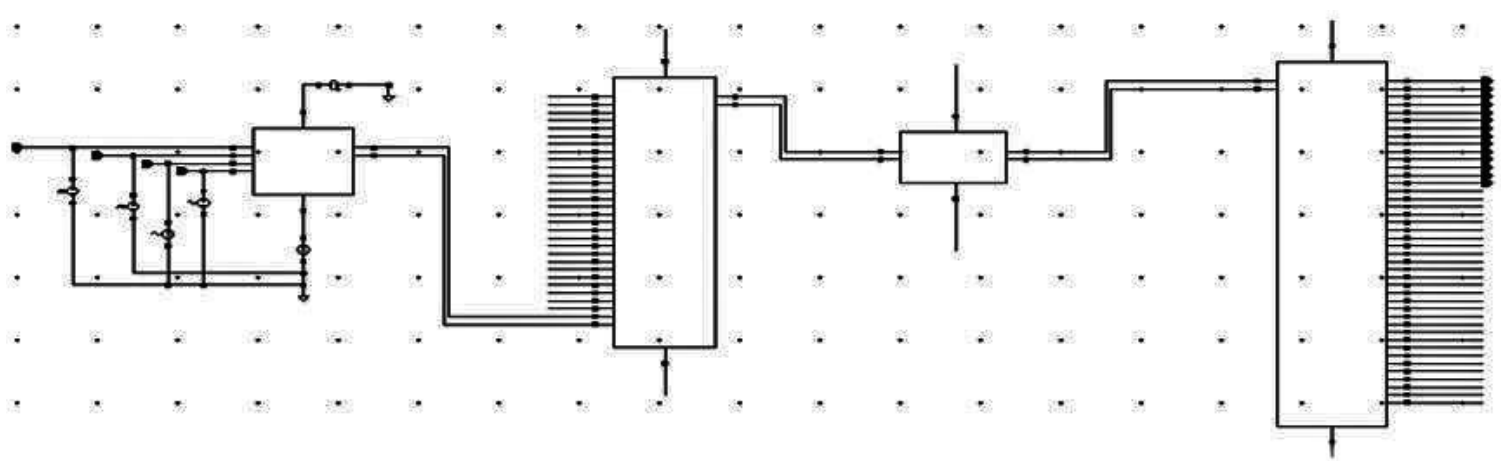

Fig. 12. Complete Schematic of 14-bit SAR ADC

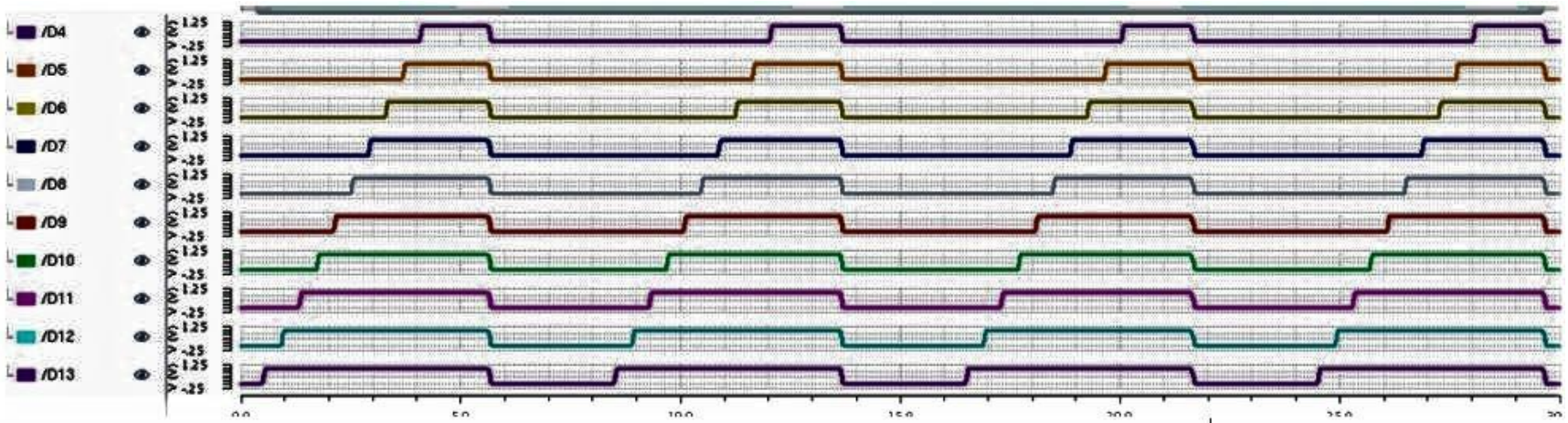

Fig. 13. Transient Response of 14-bit SAR ADC

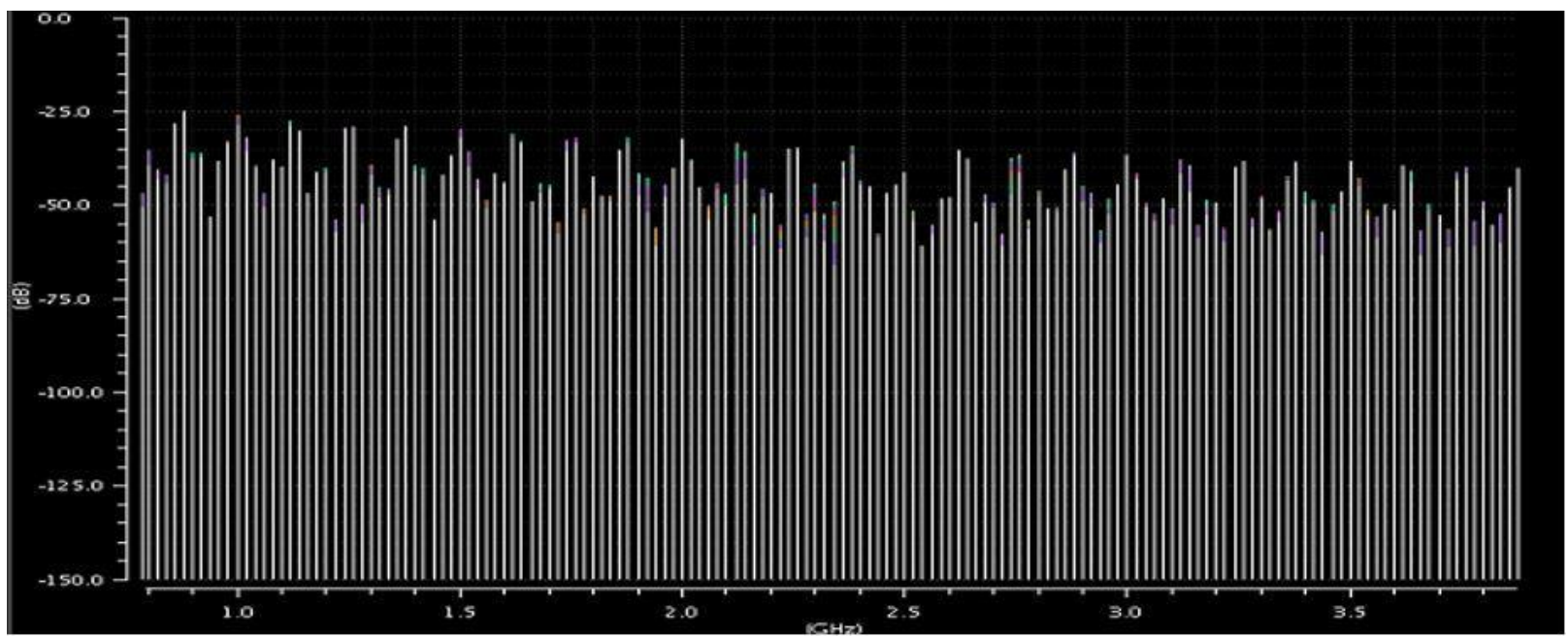

Fig. 14. FFT Spectrum of SAR ADC with 125MS/s Sampling Frequency

\section{RESULTS AND DISCUSSIONS}

The proposed work includes the development of a HighlyRobust-Dual-Split-Array-Three-Section (DSATS) capacitor DAC (DSATS-CDAC), which addresses the need for a robustDAC design for a stable and energy efficient SAR-ADC structure. The (DSATS-CDAC) architecture allows for two split segments, unlike typical capacitive DAC models. This is the only proposed ADC converter that uses an optimized DAC array in terms of unit capacitance to limit the effects of capacitive mismatch and parasitic capacitance, which reduces both energy consumption from $50 \mathrm{fF}$ to $8 \mathrm{fF}$ and the total capacitance of the DAC from $824 \mathrm{pF}$ to $824 \mathrm{fF}$ when compared to the conventional CDAC, which saves a large amount of area.
The power consumption of the SAR-ADC is $42.8 \mathrm{~mW}$ measured as a function of sampling rate, which ranged from 10MSps to $125 \mathrm{MSps}$, with the supply voltage of $1 \mathrm{~V}$. To run the simulation, the sampling rate was set to $125 \mathrm{MSps}$, and the SAR - ADC input supply was set to $1 \mathrm{~V}$. The switching energy consumed by the proposed DAC design is $8 \mathrm{fJ}$. In addition, the comparator dispersion delay is as small as $250 \mathrm{ps}$. The Fig. 14 shows the FFT-Spectrum at sampling frequency $125 \mathrm{MSps}$ with input sine wave for a frequency of $96 \mathrm{KHz}$. Table 4 summarizes the performance of the implemented SAR ADC converter when compared to contemporary state-of-the-art SAR-ADCs. With 90nm CMOS technology the sampling frequency is increased from $1 \mathrm{kSps}$ to $125 \mathrm{MSps}$ where the proposed ADC can be used 
for high speed, with bootstrap and differential sampling there is a reduced distortion and improved SNR from $55 \mathrm{~dB}$ to $81 \mathrm{~dB}$. DNL and INL in LSB as functions of the converter's output level code. For 14-bit resolution, The DNL measured at $125 \mathrm{MSps}$ is $\pm 0.16 \mathrm{LSB}$, with an INL of $\pm 0.16 \mathrm{LSB}$. The DNL/INL values are basically an indication of the performance related to the capacitance mismatch in the (DSATS-CDAC) architecture, the DNL/INL values obtained are less than 1LSB, and the proposed DAC can accurately resolve up to 13.16 bits for a proposed 14-bit resolution is one of the key achieved parameter. The figure of merit (FOM) of the proposed ADC is $37.3 \mathrm{fJ} /$ conversion-step at $125 \mathrm{Ms} / \mathrm{s}$ and $1 \mathrm{~V}$ supply which is better than other architectures as shown in Table III. The parameters obtained by the proposed converter are comparable to those obtained by previous medium-resolution converters.

TABLE III. COMPARISON WITH OTHER WORKS

\begin{tabular}{|c|c|c|c|c|c|c|}
\hline & {$[30]$} & {$[7]$} & {$[15]$} & {$[24]$} & {$[25]$} & Proposed Work \\
\hline Process Technology (nm) & 65 & 130 & 180 & 90 & 65 & 90 \\
\hline Resolution (bits) & 10 & 12 & 11 & 10 & 14 & 14 \\
\hline Sampling Rate (MS/s) & $160 \mathrm{M}$ & $1 \mathrm{~K}$ & $10 \mathrm{~K}$ & $100 \mathrm{~K}$ & $10 \mathrm{~K}$ & $125 \mathrm{M}$ \\
\hline Supply Voltage (V) & 1.2 & 1 & 0.75 & 1 & 0.8 & $1 \mathrm{~V}$ \\
\hline Power Consumption (W) & $2 \mathrm{~m}$ & $110 \mathrm{n}$ & $250 \mathrm{n}$ & $0.5 \mu$ & $1.98 \mu$ & $42.82 \mathrm{~m}$ \\
\hline ENOB & 8.9 & 10.47 & 9.76 & 8.5 & 12.5 & 13.16 \\
\hline FOM (fJ/conv) & 25.4 & 76 & 28.8 & 9.76 & 34.2 & 37.43 \\
\hline SNR (dB) & 55.6 & 64.8 & 60.5 & - & 77 & 81 \\
\hline DNL (LSB) & $+0.47 /-0.75$ & $+0.35 /-0.41$ & $+0.6 /-0.37$ & - & $+2.28 /-0.90$ & \pm 0.16 \\
\hline INL (LSB) & $+0.93 /-0.97$ & $+0.60 /-0.74$ & $+0.94 /-0.89$ & - & $+2.22 /-2.26$ & \pm 0.16 \\
\hline
\end{tabular}

\section{CONCLUSION}

A unique enhanced-linearity type 14-bit Asynchronous SAR ADC is suggested, featuring a low noise comparator with a switching latency of 250ps and differential sampling topology to improve SNR to $81 \mathrm{~dB}$ and minimize the ADC's energy consumption. DSATS-CDAC is proposed with multiplexerbased switching to save $59.76 \%$ of the area and make the system more efficient. An improved SAR logic is also proposed to reduce the delay even further. The suggested ADC is simulated using a 90nm CMOS technology with a $1 \mathrm{~V}$ supply, and the results show that it performs better than earlier approaches. Finally, the ADC achieves a sampling rate of $125 \mathrm{MS} / \mathrm{s}$ with ENOB of 13.16, FOM of $37.43 \mathrm{fJ} /$ Conversion-step, and Differential and Integral nonlinearity of $0.16 \mathrm{LSB}$. The efficient switching of the DSATS-CDAC suggests that the proposed ADC is monotonic with linear characteristics for a high resolution of 14 bits, based on these findings, the suggested $\mathrm{ADC}$ is appropriate for high-speed real-time and biomedical applications.

\section{REFERENCES}

[1] Zha, Y.; Zahnd, L.; Deng, J.; Ru eux, D.; Badami, K.; Mavrogordatos, T.; Matsuo, Y.; Emery, S. An Untrimmed PVT-Robust 12-bit 1-MS/s SAR ADC IP in $55 \mathrm{~nm}$ Deeply Depleted Channel CMOS Process. In Proceedings of the 2019 IEEE Asian Solid-State Circuits Conference (ASSCC), Macau, Macao, 4-6 November 2019; pp. 13-16.

[2] Verma, D.; Shehzad, K.; Khan, D.; Ain, Q.U.; Kim, S.J.; Lee, D.; Pu, Y.; Lee, M.; Hwang, K.C.; Yang, Y.; et al.A Design of 8 fJ/Conversion-Step 10-bit $8 \mathrm{MS} / \mathrm{s}$ Low Power Asynchronous SAR ADC for IEEE 802.15.1 IoTSensor Based Applications. IEEE Access. 2020, 8, 85869-85879.

[3] Shehzad, K.; Verma, D.; Khan, D.; Ain, Q.U.; Basim, M.; Kim, S.J.; Pu, Y.: Hwang, K.C.; Yang, Y.; Lee, K.-Y.Design of a Low Power 10-b 8$\mathrm{M}$ SS/s Asynchronous SAR ADC with On-Chip Reference Voltage. Gener. Electron.2020, 9, 872 .

[4] Q. Fan and J. Chen, "A 500-MS/s 13-Bit SAR-Assisted Time-Interleaved Digital-Slope ADC," 2019 IEEE International Symposium on Circuits and Systems (ISCAS), Sapporo, Japan, 2019, pp. 1-5, doi: 10.1109/ISCAS.2019.8702383.
[5] K. Kuo, "A 10-bit $250 \mathrm{MS} / \mathrm{s}$ Binary Search and Two channel SAR ADC by a two-bit per Conversion with Error Tolerance Ability," 2019 International SoC Design Conference (ISOCC), Jeju, Korea (South), 2019, pp. 1-2, doi: 10.1109/ISOCC47750.2019.9027763.

[6] Polineni, S., Bhat, M.S. \& Rekha, S. A Switched Capacitor-Based SAR ADC Employing a Passive Reference Charge Sharing and Charge Accumulation Technique. Circuits Syst Signal Process (2020), doi.org/10.1007/s00034-020-01437-3.

[7] W. Mao, Y. Li, C. Heng and Y. Lian, "A Low Power 12-bit 1-kS/s SAR ADC for Biomedical Signal Processing," in IEEE Transactions on Circuits and Systems I: Regular Papers, vol. 66, no. 2, pp. 477-488, Feb. 2019, doi: 10.1109/TCSI.2018.2859837.

[8] B. Chen, M. Maddox, M. C. W. Coln, Y. Lu and L. D. Fernando, "Precision Passive-Charge-Sharing SAR' ADC: Analysis, Design, and Measurement Results," in IEEE Journal of Solid-State Circuits, vol. 53, no. 5, pp. 1481-1492, May 2018, doi: 10.1109/JSSC.2018.2793558

[9] M. Kulkarni, C. Parikh and S. Sen, "A Systematic Approach to Determining the Weights of the Capacitors in the DAC of a Non-binary Redundant SAR ADCs," 2018 31st International Conference on VLSI Design and 2018 17th International Conference on Embedded Systems (VLSID), Pune, 2018, pp. 1-6, doi: 10.1109/VLSID.2018.28.

[10] S. Kim and K. Kwon, "A hybrid ADC combining capacitive DAC-based multi-bit/cycle SAR ADC with flash ADC," 2016 International Conference on Electronics, Information, and Communications (ICEIC), Da Nang, 2016, pp. 1-4, doi: 0.1109/ELÍNFOCOM.2016.7562950.

[11] W. Kim et al., "A 0.6 V 12 b 10 MS/s Low-Noise Asynchronous SARAssisted Time-Interleaved SAR (SATI-SAR) ADC," in IEEE Journal of Solid-State Circuits, vol. 51, no. 8, pp. 1826-1839, Aug. 2016, doi: 10.1109/JSSC.2016.2563780

[12] Chen, Y. Zhang, J. L. Ceballos and G. C. Temes, "Noise-shaping SAR ADC using three capacitors," in Electronics Letters, vol. 49, no. 3, pp. 182-184, 31 Jan. 2013, doi: 10.1049/el.2012.4204.

[13] D. Zhang, A. Bhide and A. Alvandpour, "A 53-nW 9.12-ENOB 1-kS/s SAR ADC in 0.13-um CMOS for medical implant devices." 2011 Proceedings of the ESSCIRC (ESSCIRC), Helsink1, 2011, pp. 467-470, doi: 10.1109/ESSCIRC.2011.6045008.

[14] C. Liu, M. Huang and Y. Tu, "A 12 bit 100 MS/s SAR-Assisted DigitalSlope ADC," in IEEE Journal of Solid-State Circuits, vol. 51, no. 12, pp. 2941-2950, Dec. 2016, doi: 10.1109/JSSC.2016.2591822.

[15] M. Sadollahi, K. Hamashita, K. Sobue and G. C. Temes, "An 11-Bit 250$\mathrm{nW} 10-\mathrm{kS} / \mathrm{s}$ SAR ADC With Doubled Input Range for Biomedical Applications," in IEEE Transactions on Circuits and Systems I: Regular Papers, vol. 65, no. 1, pp. 61-73, Jan. 2018, doi: 10.1109/TCSI.2017.2712066

[16] İ. Özkaya, Ç. Gürleyük, A. Ergül, A. Akkaya and D. Y. Aksın, "A 50V input range 14bit $250 \mathrm{kS} / \mathrm{s}$ ADC with $97.8 \mathrm{~dB}$ SFDR and $80.2 \mathrm{~dB}$ SNR," ESSCIRC 2014 - 40th European Solid State Circuits Conference (ESSCIRC), Venice Lido, 2014, pp. 71-74, doi: 10.1109/ESSCIRC.2014.6942024.

[17] K. Kurihara, K. Kobayashi, M. Uemori, M. Arai and H. Kobayashi, "Fundamental design consideraion of sampling circuit," 2016 
International Symposium on VLSI Design, Automation and Test (VLSIDAT), Hsinchu, 2016, pp. 1-4

[18] Xinquan Lai, Donglai Xu, Lvshun Hu and Hongyi Wang, "A high precision and low noise S/H circuit design for video signal sampling," Proceedings of the 200411 th IEEE International Conference on Electronics, Circuits and Systems, 2004. ICECS 2004., Tel Aviv, Israel, 2004, pp. 310-313

[19] T. B. Nazzal and S. A. Mahmoud, "Low-power bootstrapped sample and hold circuit for analog-to-digital converters," 2016 IEEE 59th International Midwest Symposium on Circuits and Systems (MWSCAS), Abu Dhabi, 2016, pp. 1-4

[20] Prakruthi T.G and S. Yellampalli, "Design and implementation of sample and hold circuit in 180nm CMOS technology," 2015 Internationa Conference on Advances in Computing. Communications and Informatics (ICACCI), Kochi, 2015, pp. 1148-1151.

[21] Mohammadi and M. Chahardori, "A Low-Power, Bootstrapped Sample and Hold Circuit with Extended Input Ranged for Analog-to-Digital Converters in CMOS $0.18 \mu \mathrm{m}, " 2018$ 15th International Conference on Synthesis, Modeling, Analysis and Simulation Methods and Applications to Circuit Design (SMACD), Prague, 2018, pp. 269-272

[22] S. A. Mahmoud and T. B. Nazzal, "Sample and hold circuits for lowfrequency signals in analog-to-digital converter," 2015 International Conference on Information and Communication Technology Research (ICTRC), Abu Dhabi, 2015, pp. 36-39.

[23] S. Lee, A. P. Chandrakasan and H. Lee, "A 1 GS/s 10b 18.9 mW TimeInterleaved SAR ADC With Background Timing Skew Calibration," Dec. 2014, doi: 10.1109/JSSC.2014.236285i.

[24] T. Yousefi, A. Dabbaghian and M. Yavari, "An Energy-Efficient DAC Switching Method for SAR ADCs," in IEEE Transactions on Circuits and Systems II: Express Briefs, vol. 65 , no. 1, pp. 41-45, Jan. 2018, doi: 10.1109/TCSII.2017.2676048.

[25] Savitha, M. and Reddy, R.V.S. (2019), "Dual split-three segment capacitor array design based successive approximation ADC for Io-T ecosystem", Integration, Vol. 69, pp. 279-288.

[26] C. Liu, S. Chang, G. Huang and Y. Lin, "A 10-bit 50-MS/s SAR ADC With a Monotonic Capacitor Switching Procedure," in IEEE Journal of Solid-State Circuits, vol. 45, no. 4, pp. 731-740, April 2010, doi: 10.1109/JSSC.2010.2042254.

[27] C. Li, C. Chan, Y. Zhu and R. P. Martins, "Analysis of Reference Error in High-Speed SAR ADCs With Capacitive DAC," in IEEE Transactions on Circuits and Systems I: Regular Papers, vol. 66, no. 1, pp. 82-93, Jan. 2019, doi: 10.1109/TCSI.2018.2861835.

[28] Polineni, S., Bhat, M.S. \& Rajan, A. A 10-Bit Differential Ultra-LowPower SAR ADC with an Enhanced MSB Capacitor-Split Switching Technique. Arab J Sci Eng 44, 2345-2353 (2019).

[29] Tong, X., Song, M., Chen, Y. et al. A 10-Bit 120 kS/s SAR ADC without Reset Energy for Biomedical Electronics. Circuits Syst Signal Process 38, 5411-5425 (2019)

[30] D. Xu et al., "A Linearity-Enhanced 10-Bit 160-MS/s SAR ADC With Low-Noise Comparator Technique," in IEEE Transactions on Very Large Scale Integration (VLSI) Systems, vol. 27, no. 9, pp. 1990-1997, Sept. 2019, doi: 10.1109/TVLSI.2019.2912504

[31] D. Xing, Y. Zhu, C. Chan, F. Maloberti, S. U and R. P. Martins, "Design of a High-Speed Time-Interleaved Sub-Ranging SAR ADC With Optimal Code Transfer Technique," in IEEE Transactions on Circuits and Systems I: Regular Papers, vol. 66, no. 2, pp. 489-501, Feb. 2019, doi: 10.1109/TCSI.2018.2866958.

[32] W. Xu and $\mathrm{H}$. Chen, "Designing a precision comparator for 10-bit synchronization SAR ADC," 2014 International Conference on AntiCounterfeiting, Security and Identification (ASID), Macao, 2014, pp. 14 ,

[33] G. Naveen and S. Sonoli, "Design and simulation of 10-bit SAR ADC for low power applications using $180 \mathrm{~nm}$ technology," 2016 International Conference on Electrical, Electronics, Communication, Computer and Conference on Electrical, Electronics, Communication, Computer
Optimization Techniques (ICEECCOT), Mysuru, 2016, pp. 331-335.

[34] Y. Cao, Y. Chen, Z. Ni, F. Ye and J. Ren, "An 11b 80MS/s SAR ADC With Speed-Enhanced SAR Logic and High-Linearity CDAC," 2018 IEEE Asia Pacific Conference on Circuits and Systems (APCCAS), Chengdu, 2018, pp. 18-21

[35] Y. Yu, F. Huang and C. Wang, "A 1V 10-bit 500KS/s energy-efficient SAR ADC using Master-Slave DAC technique in 180nm Design, Automation and Test, Hsinchu, 2014, pp. 1-4

[36] J. Wen, P. Chang, J. Huang and W. Lai, "Chip design of a 12-bit 5MS/s fully differential SAR ADC with resistor-capacitor array DAC technique for wireless application," 2015 IEEE International Conference on Signal Processing, Communications and Computing (ICSPCC), Ningbo, 2015, pp. 1-4.

[37] Verma, D.; Shehzad, K.; Khan, D.; Ain, Q.U.; Kim, S.J.; Lee, D.; Pu, Y.G.; Lee, M.; Hwang, K.C.; Y̧ang, Y.; et al. A Design of 8 fJ/Conversion-Step 10-bit 8 MS/s Low Power Asynchronous SAR ADC for IEEE 802.15.1 IoT Sensor Based Applications. IEEE Access 2020, 8,
85869-85879.

[38] Shehzad, K.; Verma, D.; Khan, D.; Ain, Q.U.; Basim, M.; Kim, S.J.; Pu, Y.; Hwang, K.C.; Yang, Y.; Lee, K.-Y. Design of a Low Power 10-b 8$\mathrm{MS} / \mathrm{s}$ Asynchronous SAR ADC with On-Chip Reference Voltage Generator. Electronics 2020, 9, 872.

[39] Verma, D.; Shehzad, K.; Khan, D.; Kim, S.J.; Pu, Y.G.; Yoo, S.-S.; Hwang, K.C.; Yang, Y.; Lee, K.-Y. A Design of Low-Power 10-bit 19, 1100 .
[40] D. S. Marathe and U. P. Khot, "A 10-Bit 10-Ms/S 5.72 nW Mixed SAR Logic for ADC Used in Wireless Sensor Node," 2019 International
Conference on Nascent Technologies in Engineering (ICNTE), Navi Mumbai, India, 2019, pp. 1-6

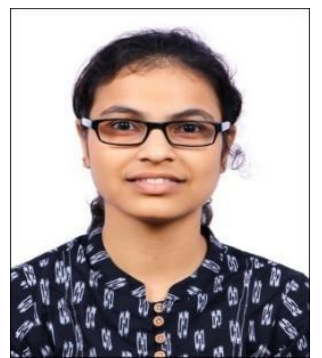

CHAYA SHETTY obtained her graduation in BE, Electronics \& Communication Engineering from NMAM Institute of Technology, Nitte in 2018 and is currently pursuing M.Tech in VLSI and Embedded Systems at Ramaiah Institute of Technology, Bangalore.

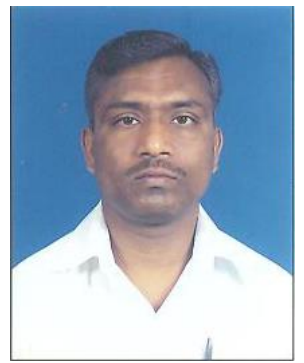

M. NAGABUSHANAM obtained his B.E. degree from Bangalore University in Electronics Engineering in 1997, M.Tech in Electronics Engineering from Visvesvaraya Technological University in 2003 \& obtained Ph.D in VLSI from Anna University, Chennai in 2017. He joined Department of Electronics \& Communication Engineering, M.S. Ramaiah Institute of Technology in 2003 as Assistant Professor. He has over 19 years of teaching experience in Analog Electronics, VLSI, and Advanced mixed signal design, linear integrated circuits. Microelectronics, CMOS VLSI design, solid state devices, optical fiber communications. His area of specialization is VLSI, Image processing, mixed signal design. He is a recognized reviewer for few reputed journals and has published more than 20 research papers in National and International Journals. He is also a visiting professor at Manipal University, Bangalore University, Birla institute of technology \& science. He is a life member ISTE since 2010 and member IAENG since 2019.

2. VENKATESH NUTHAN PRASAD completed his (a)

If 1 graduation with a B.E. degree in Electronics and Communication Engineering from Visvesvaraya Technological University in 2003, Masters in Digital Electronics and Communication from the same university during 2006. Currently he is pursuing his Ph.D in Antenna design. His areas of interest are Information Theory and Coding, Analog, Digital circuits Design, Mixed signal designs 
Contribution of individual authors to the creation of a scientific article (ghostwriting policy)

Author Contributions: Please, indicate the role and the contribution of each author:

1. Chaya Shetty M.tech student did the study on data converters and Designed SAR ADC converter and carried out simulation, executed and prepared original draft.

2. Dr. M. Nagabushanam has Designed the complete Architecture with specifications and derived (W/L) for the architecture in $90 \mathrm{~nm}$ CMOS technology and is responsible for review and editing

3. Venkatesh. Nuthan Prasad was involved in reducing bottlenecks in design during simulation and also responsible for editing

Sources of funding for research presented in a scientific article or scientific article itself

Report potential sources of funding if there is any

No source of Funding
Creative Commons Attribution License 4.0 (Attribution 4.0 International, CC BY 4.0)

This article is published under the terms of the Creative Commons Attribution License 4.0

https://creativecommons.org/licenses/by/4.0/deed.en US 